\title{
DEMONSTRATIVO DE VANTAGENS ECONÔMICAS E DE EMISSÕES DE GASES DE EFEITO ESTUFA NA OPÇÃO POR ETANOL NOS ABASTECIMENTOS DE VEÍCULOS FLEX-FUEL
}

\author{
Cláudio Silva Graminho ${ }^{1 *}$, Alexandre Dullius² \\ 1*Universidade Federal do Paraná, Curitiba, Paraná, Brasil, claudio.graminho@copel.com \\ ${ }^{2}$ Instituto Federal do Paraná, Campus Paranaguá, Paranaguá, Paraná, Brasil, alexandre.dullius@ifpr.edu.br
}

\begin{abstract}
Resumo
A diferença entre o preço do combustível no abastecimento de uma frota de veículos flex-fuel com etanol ou com gasolina não deve ser o principal quesito na escolha do combustível empregado. Utilizando os dados de abastecimento de um conjunto de um pool de veículos flex-fuel de uma grande empresa durante todo o ano de 2014, onde se utilizou majoritariamente a opção da gasolina como combustível. Este trabalho propõe uma metodologia para o cálculo da diferença entre os preços no abastecimento e entre a quantidade de emissões de gases de efeito estufa gerados tanto pelo uso misto de combustíveis, quanto pelo uso exclusivo do etanol hidratado estimado para se percorrer a mesma distância. Também se estabeleceu uma relação entre a diferença de preços por litro dos dois combustíveis e a economia gerada. Por fim, conclui-se que há realmente vantagens financeiras e ambientais na adoção da preferência ao biocombustível. Com o estudo, acredita-se na contribuição com as discussões voltadas à temática em foco e na orientação na tomada de decisões políticas e econômicas voltadas à utilização de etanol, bem como para políticas de sustentabilidade.
\end{abstract}

Palavras-chave: Combustíveis; Biocombustível; GEE; Eficiência energética; Gasolina.

\begin{abstract}
Statement of economic benefits and greenhouse gas emissions in option for ethanol in supplies of flex-fuel vehicles. When choosing which fuel to use on a fleet of flex-fuel vehicles, whether applying ethanol or gasoline, the difference in cost shouldn't be the main point to consider. Using the data collected from fueling a major company's group of flex-fuel vehicles throughout 2014, in which gasoline was used as the main fuel option, this research proposes a methodology to calculate the difference between the financial costs of fueling and the emission of greenhouse gases, be it from mixing fuels or exclusively using an amount estimated of hydrated ethanol. A connection was also established regarding the difference in price per liter of both fuels and the savings generated. Finally, it can be concluded that there truly are financial and environmental advantages in adopting biofuel as a preference. With this study, it is possible to believe in the contribution to the discussions of the theme in focus, as well as the guidance in taking political and economical decisions regarding the use of ethanol, along with sustainability policies.
\end{abstract}

Keywords: Fuel; Biofuel; GHG; Energy efficiency; Gasoline.

\section{INTRODUÇÃO}

Apesar das reservas provadas de petróleo permanecerem estáveis, a produção mundial de combustíveis fósseis ainda demonstra crescimento, com uma taxa de 0,64\% entre o ano 2012 e 2013. Nesse mesmo período, o consumo de combustíveis aumentou 1,16\%, quase o dobro da taxa de produção (ANP, 2014), prenunciando uma escassez a médio e longo prazos. Contudo, a questão econômica de recursos é reforçada pela insustentabilidade ambiental da opção fóssil, historicamente mais utilizada. Há praticamente um consenso sobre as questões das mudanças climáticas, inclusive quanto às causas antropológicas dessas mudanças, enfatizadas e reafirmadas pelos seguidos relatórios do Painel Intergovernamental sobre Mudanças Climáticas (IPCC, 2014).

No quinto relatório do IPCC (2014), é afirmado que são necessárias mais ações de corte das emissões de gases de efeito estufa (GEE) para limitar o aquecimento do planeta a $2^{\circ} \mathrm{C}$ até 2100 . Segundo os cientistas do IPCC, é preciso abandonar os combustíveis fósseis e utilizar fontes mais limpas a fim de reduzir estas emissões que poderiam provocar um aumento da temperatura do planeta 
entre $3,7^{\circ} \mathrm{C}$ e $4,8^{\circ} \mathrm{C}$ antes de 2100 , o que seria um nível catastrófico.

Numa análise do consumo energético e de emissões de $\mathrm{CO} 2$ no setor de transportes brasileiro, Andrade e Mattei (2011) observam que a principal estrutura de transportes do país é o transporte rodoviário, com $61 \%$ do modal utilizado, atingindo o patamar de $96 \%$ quando se trata de transporte de passageiros. O estudo aponta que esse setor é a segunda maior fonte de emissões de GEE no Brasil, com um consumo energético acima da média mundial, ressaltando a importância da necessidade de um correto dimensionamento das emissões oriundas da frota nacional.

Uma dessas alternativas renováveis proporcionou uma posição de vanguarda ao país já na década de 1970: o etanol originário da cana-de-açúcar. Segundo a Empresa Brasileira de Pesquisa Agropecuária, o biocombustível apresenta o melhor balanço energético do mundo, pois para cada unidade de energia fóssil são armazenas 9,3 unidades de energia (COLLARES, 2010). O etanol apresentou um grande crescimento de uso logo após o seu lançamento em 1975, alavancado pela crise do petróleo. Após essa fase inicial, houve um grande período de crise, devido ao baixo preço do petróleo e ao excesso de oferta do etanol (FILHO; RAMOS, 2006).

As vendas do biocombustível apresentaram novamente forte crescimento a partir da entrada dos veículos flex-fuel em 2003, impulsionado pelo o aumento da renda e do consumo e da substituição da gasolina pelo etanol. A crise de 2008, no entanto, afetou significativamente os produtores de canade-açúcar, influenciando seus preços. A partir de 2009, o etanol perdeu a competitividade em termos de preço versus eficiência, mesmo sendo considerado um biocombustível avançado (ANP, 2013). Apesar do retorno gradual, ele ainda está longe de ser considerado como preferência na hora do abastecimento, devido à falsa associação do seu poder calorífico com sua eficiência no cômputo geral. De qualquer maneira, a gasolina comum, do Tipo C, é acrescida de etanol anidro. Atualmente a taxa de mistura é de $27 \%$ (CIMA, 2015), porém a mistura era de 25\% no ano base de 2014 (MAPA, 2013).

Comumente é considerado, tanto pelas empresas quanto pelo consumidor em geral, apenas a questão financeira na escolha do combustível. É senso comum considerar uma proporção de $70 \%$ entre o preço do etanol e da gasolina na bomba de combustível como valor máximo em que sua escolha é mais rentável. Essa taxa estimada corresponde ao menor poder calorífico do biocombustível do bagaço da cana-de-açúcar (SANTOS, 2008), porém o índice de eficiência depende de muitos outros fatores.

Assim, este trabalho leva em consideração essa diversidade de variáveis, como as diferenças nas eficiências de motores e a variação de preços dos dois combustíveis, e busca agregar a questão sustentável como incentivo no momento do abastecimento. Com isso, objetivou-se estabelecer possíveis ganhos econômicos e ambientais no abastecimento de veículos flex-fuel com a utilização exclusiva de etanol, ao invés da combinação de etanol e gasolina, através da relação entre estes dois combustíveis.

\section{MATERIAL E MÉTODOS}

\section{Local de estudo}

A empresa que proveu os dados para este estudo tem uma política ativa de sustentabilidade. Nos últimos anos, houve um investimento na substituição gradativa de veículos movidos à gasolina por veículos flex-fuel, como forma de incentivar o uso de etanol. Porém, como levantamento preliminar para este trabalho, constatou-se que $91 \%$ dos abastecimentos daqueles veículos foram realizados utilizando-se gasolina. Assim, contemplou-se o uso dos veículos utilizados dessa frota no estado do Paraná. A empresa contava, ao final de 2014, com cerca de 8.600 empregados.

\section{Abordagem de estudo}

O ponto de partida deste trabalho é o histórico de abastecimento da frota da de veículos da empresa 3.869 veículos em 31 de dezembro de 2014. Desses, foram selecionados os veículos leves, segundo resolução do Conselho Nacional do Meio Ambiente (CONAMA, 1995), com massa total máxima até $3.856 \mathrm{~kg}$ e massa do veículo até $2.720 \mathrm{~kg}$.

Do total de veículos leves, 690 foram flex-fuel com anos de fabricação de 2004 a 2014. Contudo, foram desconsiderados os veículos de fabricados nos anos de 2004 e 2005, pois além da dificuldade na estimativa de rendimento, esses já foram vendidos no ano corrente, visto que atingiram a marca de 10 anos de meta de utilização pela companhia.

Por fim, os modelos foram consultados no Programa Brasileiro de Etiquetagem Veicular - PBE, do Instituto Nacional de Metrologia, Qualidade e Tecnologia (INMETRO, 2015), e selecionados aqueles 
que receberam a Etiqueta Nacional de Conservação de Energia, com seus índices de eficiência estabelecidos em laboratório. Assim, a base de estudo foi concentrada em 508 veículos, distribuídos em 29 modelos (Tabela 1).

Tabela 1. Modelos automotivos da frota flex-fuel homologados pelo INMETRO

\begin{tabular}{ccccc}
\hline Ano de fabricação & Modelo & Motorização Número de veículos & Ano da etiquetagem no PBE \\
\hline 2006 & Kombi Furgão & 1.4 & 3 & 2011 \\
2006 & Saveiro & 1.6 & 63 & 2010 \\
2007 & Kangoo AUT Express & 1.6 & 15 & 2012 \\
2007 & Kangoo RL 1.6 Express & 1.6 & 29 & 2012 \\
2007 & Kombi Standard & 1.4 & 1 & 2011 \\
2007 & Uno Mille Fire & 1.0 & 48 & 2009 \\
2007 & Parati 1.6 Plus & 1.6 & 20 & $2010^{*}$ \\
2007 & Saveiro & 1.6 & 63 & 2010 \\
2008 & Gol Geração 5 & 1.6 & 2 & 2009 \\
2009 & Dobló Cargo & 1.8 & 16 & 2012 \\
2009 & Gol Geração 4 & 1.0 & 32 & 2009 \\
2009 & Parati 1.6 Plus & 1.6 & 11 & $2010^{*}$ \\
2009 & Saveiro & 1.6 & 30 & 2010 \\
2010 & Dobló Cargo Essence & 1.8 & 1 & 2012 \\
2010 & Parati 1.6 Plus & 1.6 & 2 & $2010^{*}$ \\
2011 & Dobló Attractiv & 1.4 & 4 & 2012 \\
2011 & Dobló Cargo & 1.4 & 7 & 2012 \\
2011 & Fiesta & 1.0 & 3 & $2011^{* *}$ \\
2011 & Kombi Standard & 1.4 & 4 & 2911 \\
2011 & Palio Weekend ELX & 1.4 & 47 & $2011^{* * *}$ \\
2011 & Parati 1.6 Plus & 1.6 & 2 & $2010^{*}$ \\
2011 & Strada Fire & 1.4 & 19 & 2011 \\
2012 & Dobló Attractiv & 1.4 & 2 & 2012 \\
2012 & Palio Weekend ELX & 1.4 & 15 & $2011^{* * *}$ \\
2012 & Strada Fire & 1.4 & 17 & 2012 \\
2012 & Strada Locker Adventure & 1.8 & 15 & 2012 \\
2012 & Uno Attractive Evo & 1.4 & 33 & 2012 \\
2014 & ETIOS HB X & 1.3 & 1 & 2014 \\
2014 & Sandero Expression & 1.6 & 3 & 2015 \\
\hline FBE & 2015 & &
\end{tabular}

Fonte: PBE (2015).

Em que: * Média dos motores Volkswagen Flex 1.6 de 2010; ${ }^{* *}$ Médias dos motores Ford Flex 1.0 de 2011; e *** Média dos motores Fiat 310A2011 de 2011.

A metodologia do trabalho foi um comparativo de cenários entre o abastecimento com uso misto de combustíveis (Cenário 1) e o estimado com uso exclusivo de etanol hidratado (Cenário 2). Em seguida, foram calculados os valores pagos nos abastecimentos por distância percorrida e as emissões de GEE para os abastecimentos ocorridos em 2014. Esses forneceram a linha base para a comparação e, de posse dos valores discriminados por mês e por modelo de veículo, foi possível utilizar o método Bottom Up proposto pelo GHG Protocol Brasil (GHG, 2014). Com os valores estimados de distância executada pela frota, foi calculada a quantidade de etanol hidratado necessária para se percorrer a mesma quilometragem. Com isso, estimam-se os valores pagos e as emissões para o cenário do uso único do biocombustível (Cenário 2).

\section{Fonte de dados}

Os dados para este trabalho foram extraídos mediante solicitação de pesquisas parametrizadas. Eles contemplaram, além das informações do momento do abastecimento, as relativas à marca, modelo, ano de fabricação e motorização do veículo utilizado. A Etiqueta Nacional de Conservação de Energia, criada pelo PBE em 2009, visa permitir a comparação da eficiência energética dos veículos de uma mesma categoria. Ela é afixada de forma voluntária nos modelos participantes e as tabelas publicadas no INMETRO. O PBE informa os valores de referência da quilometragem por litro, na cidade e na estrada, com diferentes combustíveis, além da classificação do veículo quanto à eficiência energética em sua categoria (INMETRO, 2015).

Uma vez que muitas variáveis influenciam no desempenho dos motores fora do laboratório, como o perfil do condutor, as condições climáticas, os padrões de tráfego, o uso de acessórios 
(em especial do ar condicionado), as cargas impostas ao veículo, a tipologia e conservação do terreno e a pressão dos pneus, optou-se por comparar os desempenhos por meio de dados laboratoriais, de acordo com os padrões da NBR 7024 (INMETRO, 2010). Os dados de rendimentos dos modelos em estudo são apresentados na Tabela 2.

Tabela 2. Rendimentos dos modelos flex-fuel para os dois combustíveis aferidos pelo INMETRO

\begin{tabular}{|c|c|c|c|c|c|}
\hline \multirow{3}{*}{ Ano da frota } & \multirow{3}{*}{ Modelo } & \multicolumn{4}{|c|}{ Rendimento (km/L) } \\
\hline & & \multicolumn{2}{|c|}{ Etanol } & \multicolumn{2}{|c|}{ Gasolina } \\
\hline & & Cidade & Estrada & Cidade & Estrada \\
\hline 2006 & Kombi Furgão & 5,7 & 8,2 & 5,7 & 8,6 \\
\hline 2006 & Saveiro & 6,7 & 8,3 & 9,9 & 12,3 \\
\hline 2007 & Kangoo AUT Express & 6,1 & 9,0 & 7,4 & 10,9 \\
\hline 2007 & Kangoo RL 1.6 Expr & 6,1 & 9,0 & 7,4 & 10,9 \\
\hline 2007 & Kombi Standard & 5,7 & 8,2 & 5,7 & 8,6 \\
\hline 2007 & Uno Mille Fire & 10,8 & 15,7 & 13,2 & 19,2 \\
\hline 2007 & Parati 1.6 Plus & 9,2 & 13,5 & 13,5 & 19,6 \\
\hline 2007 & Saveiro & 6,7 & 8,3 & 9,9 & 12,3 \\
\hline 2008 & Gol Geração 5 & 9,1 & 13,2 & 13,4 & 19,3 \\
\hline 2009 & Dobló Cargo & 5,7 & 8,2 & 6,4 & 9,4 \\
\hline 2009 & Gol Geração 4 & 9,5 & 13,9 & 13,5 & 19,9 \\
\hline 2009 & Parati 1.6 Plus & 9,2 & 13,5 & 13,5 & 19,6 \\
\hline 2009 & Saveiro & 6,7 & 8,3 & 9,9 & 12,3 \\
\hline 2010 & Dobló Cargo Essence & 5,7 & 8,2 & 6,4 & 9,4 \\
\hline 2010 & Parati 1.6 Plus & 9,2 & 13,5 & 13,5 & 19,6 \\
\hline 2011 & Dobló Attractiv & 5,9 & 6,4 & 8,6 & 9,5 \\
\hline 2011 & Dobló Cargo & 5,9 & 6,4 & 8,6 & 9,5 \\
\hline 2011 & Fiesta & 8,1 & 11,6 & 9,2 & 13,5 \\
\hline 2011 & Kombi Standard & 5,7 & 8,2 & 5,7 & 8,6 \\
\hline 2011 & Palio Weekend ELX & $\begin{array}{l}0, r \\
7,1\end{array}$ & $\begin{array}{l}0,2 \\
10,5\end{array}$ & $\begin{array}{l}5,1 \\
8,0\end{array}$ & 11,8 \\
\hline 2011 & Parati 1.6 Plus & 9,2 & 13,5 & 13,5 & 19,6 \\
\hline 2011 & Strada Fire & 7,3 & 10,6 & 8,2 & 12,0 \\
\hline 2012 & Dobló Attractiv & 5,9 & 6,4 & 8,6 & 9,5 \\
\hline 2012 & Palio Weekend ELX & 7,1 & 10,5 & 8,0 & 11,8 \\
\hline 2012 & Strada Fire & 7,3 & 10,6 & 8,2 & 12,0 \\
\hline 2012 & Strada Locker Adv. & 6,5 & 9,3 & 7,3 & 10,6 \\
\hline 2012 & Uno Attractive Evo & 7,3 & 10,6 & 9,1 & 13,3 \\
\hline 2014 & ETIOS HB X & 8,5 & 9,0 & 12,5 & 13,0 \\
\hline 2014 & Sandero Expression & 7,3 & 8,7 & 10,6 & 12,5 \\
\hline
\end{tabular}

Fonte: PBE (2015).

Apesar do preço do combustível constar na planilha de abastecimento da empresa, para que este trabalho fosse mais abrangente e replicável, optou-se pela utilização da média de preços de venda ao consumidor no estado do Paraná, segregados por mês, obtida por meio do Relatório de Defesa da Concorrência, gerado pelo Sistema de Levantamento de Preços da Agência Nacional do Petróleo, Gás Natural e Biocombustíveis (ANP, 2015), com amostras de 2.460 postos consultados em média mensal. Esses valores são apresentados na Tabela 3.

Tabela 3. Médias mensais dos preços dos combustíveis nos postos do estado do Paraná

\begin{tabular}{ccccccccccccc}
\hline & \multicolumn{10}{c}{ Valor mensal (R\$) no ano de 2014 } \\
\cline { 2 - 15 } & Janeiro & Fevereiro & Março & Abril & Maio & Junho & Julho & Agosto & Setembro & Outubro & Novembro & Dezembro \\
\hline Etanol & 2,05 & 2,07 & 2,14 & 2,14 & 2,10 & 2,03 & 1,99 & 2,01 & 2,00 & 1,98 & 1,99 & 2,01 \\
Gasolina & 2,99 & 2,99 & 2,99 & 2,99 & 2,97 & 2,93 & 2,89 & 2,93 & 2,92 & 2,90 & 2,95 & 2,97 \\
\hline
\end{tabular}

Fonte: ANP (2015).

Os principais gases de efeito estufa foram o dióxido de carbono $\left(\mathrm{CO}_{2}\right)$, o metano $(\mathrm{CH} 4)$ e o óxido nitroso (N2O), em que seus fatores de emissão (FE) são listados na Tabela 4. O FE de determinado gás corresponde à quantidade emitida dele na queima de cada litro de combustível. Os valores empregados são os utilizados para o cálculo das emissões na ferramenta GHG Protocol Brasil (GHG, 2015), com base no Balanço Energético Nacional, publicado pelo Ministério de Minas e Energia. 
Tabela 4. Fatores de emissão dos principais gases de efeito estufa emitidos por combustíveis em motores flex-fuel

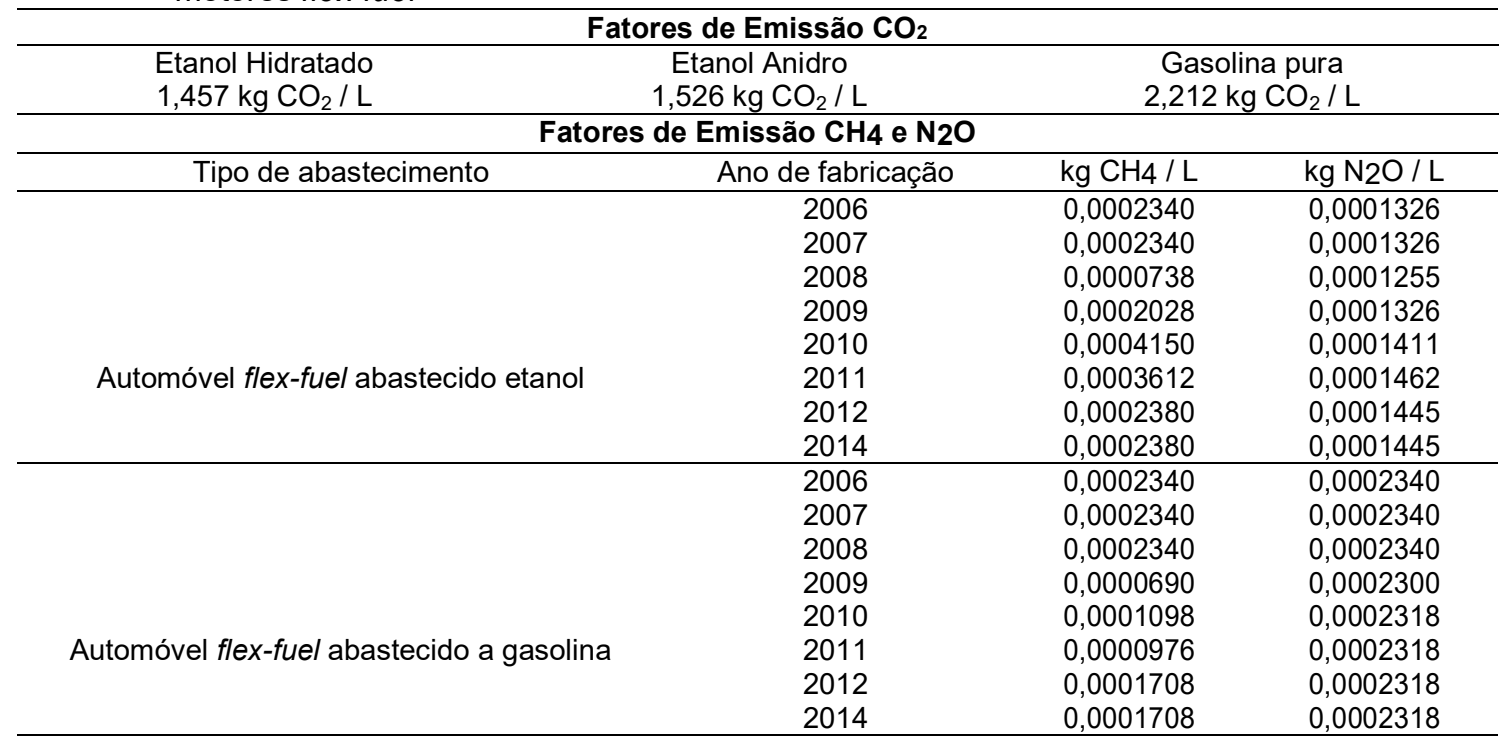

Fonte: GHG Protocol Brasil (2015).

\section{Cálculos da linha-base (Cenário 1)}

Como o uso dos automóveis é misto, tanto na estrada quanto na cidade, e não há indicações por trecho, o rendimento médio por combustível foi calculado para cada modelo por meio de média simples, a partir dos dados fornecidos pelo PBE (Tabela 2), conforme a Equação 1. Ao passo que, com os dados de rendimento de cada modelo e de abastecimento por mês, foi possível calcular a quilometragem rodada por meio da Equação 2; e com os dados de abastecimento e preço médio dos combustíveis por mês estimou-se os valores pagos com a Equação 3.

$$
\begin{gathered}
\text { ReMModeloCOMB }=(\text { ReESModeloCOMB }+ \text { ReClModeloCOMB }) / 2 \\
\mathrm{KmT}_{\text {ModeloMês }}=(\text { TAbasEModeloMês } \times \text { ReMEModelo })+(\text { TAbasGModeloMês } \times \text { ReMGModelo }) \\
\text { PgoTModeloMês = (TAbasEModeloMês } \times \text { PreçoEMês })+(\text { TAbasGModeloMês } \times \text { PreçoGMês })
\end{gathered}
$$

Em que: ReMModeloCOMB = rendimento médio do combustível para determinado modelo (km/L); ReESModeloCOMB = rendimento médio do combustível na estrada para determinado modelo ( $\mathrm{km} / \mathrm{L})$; $/ \mathrm{ReCIModeloCOMB}=$ rendimento médio do combustível na cidade para determinado modelo $(\mathrm{km} / \mathrm{L}) ; \mathrm{KmT}$ ModeloMês = quilometragem total do modelo de veículo em determinado mês $(\mathrm{km} / \mathrm{mês}) ;$ TAbasEModeloMês = total de abastecimento de etanol do modelo de veículo em determinado mês $(\mathrm{L})$; ReMEModelo = rendimento médio do etanol para o modelo $(\mathrm{km} / \mathrm{L})$; TAbasGModeloMês = total de abastecimento de gasolina do modelo de veículo em determinado mês (L); e ReMGModelo = rendimento médio da gasolina para o modelo (km/L); PgoT ModeloMês = valor total de abastecimento pago para determinado modelo em determinado mês $(\mathrm{R} \$)$; PreçoEMês $=$ preço médio de revenda do etanol hidratado no Paraná, em determinado mês $(R \$)$; e PreçoGMês = preço médio de revenda da gasolina Tipo C no Paraná, em determinado mês $(R \$)$.

A gasolina utilizada foi a do Tipo C, a qual é o resultado de uma mistura da gasolina pura (Tipo A) com etanol anidro. Para o cálculo mais preciso das emissões utilizando-se a gasolina, foi necessário que estabelecer as quantidades de cada uma das partes componentes a partir da Equação 4. Apesar de emitidos em menor quantidade, o metano e o óxido nitroso têm um potencial de aquecimento global muito maior que o gás carbônico, medido em Global Warming Potential - GWP. Assim, adotou-se o GWP igual uma unidade para $\mathrm{O} \mathrm{CO}_{2}$, ao passo que o GWP dos demais gases mostraram quanto determinado gás contribui para o efeito estufa em comparação ao $\mathrm{CO}_{2}$ (IPCC, 2007). Por definição, a partir do GWP, foi calculada a emissão equivalente em carbono de cada gás, conforme a Equação 5 . 


\author{
TAbastCOMB $=$ TAbastGC $\times$ PropCOMB \\ CO2EQ $=$ TEmis $\times$ GWP
}

Em que: TAbastCOMB = total de abastecimento do combustível componente (L); TAbastGC $=$ total de abastecimento de gasolina Tipo C (L); PropCOMB = proporção do combustível componente na mistura de $25 \%$ para o etanol anidro e de $75 \%$ para a gasolina Tipo A; CO2EQ = quantidade de emissões de $\mathrm{CO}_{2}$ equivalentes à quantidade de emissões do gás em questão; TEmis = total de emissões do gás em questão; e GWP = potencial de aquecimento global do gás em questão.

O cálculo total das emissões totais de GEE foi realizado a partir da soma da queima da biomassa e da queima do combustível fóssil, conforme as Equações 6 utilizadas na planilha de cálculo da ferramenta GHG Protocol Brasil (GHG, 2015).

$$
\begin{aligned}
\text { TGEE }=(\text { TGEECO2 }+ \text { TGEECO2EQ }) / 1.000 \\
\text { TGEECO2 }=(\text { TAbasEtH } \times \text { FECO2EtH })+(\text { TAbasEtA } \times \text { FECO2EtA }) \\
\text { TGEECO2eq }=(\text { TAbastEtH } \times \text { FECH4EtH } \times \text { GWPCH4 })+(\text { TAbastEtH } \times \text { FEN2OEtH } \times \text { GWPN2O })+ \\
+(\text { TAbastGA } \times \text { FECO2GA })+(\text { TAbastGA } \times \text { FECH4GA } \times \text { GWPCH4 })+ \\
+(\text { TAbastGA } \times \text { FEN2OGA } \times \text { GWPN2O })
\end{aligned}
$$

Em que: TGEE = total das emissões de GEE, em toneladas de $\mathrm{CO}_{2}$ equivalente; TGEECO2= emissões diretas de dióxido de carbono $(\mathrm{kg})$; TGEECO2EQ = emissões equivalentes ao dióxido de carbono $(\mathrm{kg})$; $\mathrm{TAbasEtH}=$ abastecimento total de etanol hidratado (L); TAbasEtA = porção total de etanol anidro nos abastecimentos com gasolina Tipo $C(L)$; TAbastGA = porção total de gasolina Tipo A nos abastecimentos com gasolina Tipo C (L); FECO2EtH = fator de Emissão do dióxido de carbono $(\mathrm{CO} 2)$ do etanol hidratado FECO2EtA = fator de emissão do dióxido de carbono $\left(\mathrm{CO}_{2}\right)$ do etanol anidro; $\mathrm{FECH} 4 \mathrm{EtH}$ = fator de emissão do metano $(\mathrm{CH} 4)$ do etanol hidratado; FEN2OEtH = fator de emissão do óxido nitroso $\left(\mathrm{N}_{2} \mathrm{O}\right)$ do etanol hidratado; FECO2GA = fator de emissão do dióxido de carbono $\left(\mathrm{CO}_{2}\right)$ da gasolina pura $(\mathrm{A})$; FECH4GA = fator de emissão do metano $\left(\mathrm{CH}_{4}\right)$ da gasolina pura (Tipo $\left.A\right)$; FEN2OGA = fator de emissão do óxido nitroso $(\mathrm{N} 2 \mathrm{O})$ da gasolina pura (Tipo A); GWPCH4= potencial de aquecimento global do metano $(\mathrm{CH} 4)$, i.e. 25 ; e GWPN2O = potencial de aquecimento global do óxido nitroso (N2O), i.e. 298.

\title{
Cálculos para a estimativa de uso exclusivo de etanol (Cenário 2)
}

A partir dos cálculos da quilometragem percorrida (Equação 2), dividindo-se pelo rendimento de cada veículo com etanol (Quadro 2), foi calculada a quantidade de litros desse combustível necessária para atingir a mesma quilometragem, conforme a Equação 7. Ao passo que, com a quantidade de etanol necessária por mês e com os preços mensais do etanol (Tabela 3), foram obtidos os valores caso a opção de abastecimento fosse exclusivamente com esse combustível.

Além da comparação do total geral, foi verificado qual a opção mais econômica no ato do abastecimento, por meio da Equação 8. Se a resposta da questão for afirmativa, a opção do abastecimento com o etanol foi economicamente mais vantajosa. Da mesma maneira, com a quantidade calculada de etanol necessária e a mesma metodologia utilizada para Cenário 1 , chegouse na quantidade de $\mathrm{CO} 2$ equivalente emitida no Cenário 2.

$$
\begin{gathered}
\text { TAbastEEstimado }=\text { KMREAL / ReE Modelo } \\
\text { RENDETANOL / RENDGASOLINA > PREÇOETANOL / PREÇOGASOLINA }
\end{gathered}
$$

Em que: TAbastEEstimado = quantidade necessária de etanol hidratado para percorrer a mesma quilometragem executada $(\mathrm{L}) ; \mathrm{KMREAL}=$ quilometragem realmente executada $(\mathrm{km}) ; \mathrm{ReE}$ Modelo = rendimento do etanol para o modelo $(\mathrm{km} / \mathrm{L} ; \mathrm{REND}$ (COMBUSTÍVEL) = rendimento do combustível para o veículo em estudo; e PREÇO(COMBUSTÍVEL) = preço do combustível no momento do abastecimento. 


\section{RESULTADOS E DISCUSSÃO}

\section{Quilometragem rodada}

A Tabela 5 demonstra o cálculo da quilometragem rodada a partir da somatória de abastecimentos, para o mês de janeiro, a título de exemplo.

Tabela 5. Exemplo de cálculos de quilometragem rodada para mês de janeiro/2014

\begin{tabular}{|c|c|c|c|c|c|c|c|c|}
\hline \multirow{2}{*}{$\begin{array}{l}\text { Ano da } \\
\text { frota }\end{array}$} & \multirow{2}{*}{ Modelo } & \multicolumn{2}{|c|}{ Rendimento (km/L) } & \multicolumn{2}{|c|}{ Abastecimento (L) } & \multicolumn{3}{|c|}{$\mathrm{Km}$ rodado } \\
\hline & & Etanol & Gasolina & Etanol & Gasolina & Etanol & Gasolina & Total \\
\hline 2006 & Kombi Furgão & 6,95 & 7,15 & & 62,3 & & 445,4 & 445,4 \\
\hline 2006 & Saveiro & 7,50 & 11,10 & $3.085,9$ & $6.444,7$ & $23.144,3$ & $71.536,2$ & $94.680,4$ \\
\hline 2007 & Kangoo AUT Express & 7,55 & 9,15 & 198,5 & 810,3 & $1.498,7$ & $7.414,2$ & $8.912,9$ \\
\hline 2007 & Kangoo RL 1.6 Expr. & 7,55 & 9,15 & 299,5 & $2.909,2$ & $2.261,2$ & $26.619,2$ & $28.880,4$ \\
\hline 2007 & Kombi Standard & 6,95 & 7,15 & & 57,4 & & 410,4 & 410,4 \\
\hline 2007 & Uno Mille Fire & 13,25 & 16,20 & 1248,5 & $3.883,4$ & $16.542,6$ & $62.911,1$ & $79.453,7$ \\
\hline 2007 & Parati 1.6 Plus & 11,32 & 16,55 & 834,1 & $1.149,1$ & $9.439,2$ & $19.017,6$ & $28.456,8$ \\
\hline 2007 & Saveiro & 7,50 & 11,10 & $2.522,0$ & $4.606,6$ & $18.915,0$ & $51.133,3$ & $70.048,3$ \\
\hline 2008 & Gol Geração 5 & 11,15 & 16,3 & & 84,2 & & $1.376,7$ & $1.376,7$ \\
\hline 2009 & Dobló Cargo & 6,95 & 7,90 & 122,5 & $1.227,0$ & 851,4 & $9.693,3$ & $10.544,7$ \\
\hline 2009 & Gol Geração 4 & 11,70 & 16,70 & $1.170,1$ & $2.002,8$ & $13.690,2$ & $33.446,8$ & $47.136,9$ \\
\hline 2009 & Parati 1.6 Plus & 11,32 & 16,55 & 429,1 & $1.070,1$ & $4.856,0$ & $17.710,2$ & $22.566,1$ \\
\hline 2009 & Saveiro & 7,50 & 11,10 & $1.178,3$ & $3.410,4$ & $8.837,3$ & $37.855,4$ & $46.692,7$ \\
\hline 2010 & Dobló Cargo & 6,95 & 7,90 & & 271,2 & & $2.142,5$ & $2.142,5$ \\
\hline 2010 & Parati 1.6 Plus & 11,32 & 16,55 & & 532,8 & & $8.817,8$ & $8.817,8$ \\
\hline 2011 & Dobló Attractiv & 6,15 & 9,05 & & 566,3 & & $5.125,0$ & $5.125,0$ \\
\hline 2011 & Dobló Cargo & 6,15 & 9,05 & 49,5 & $1.195,5$ & 304,4 & $10.819,3$ & $11.123,7$ \\
\hline 2011 & Fiesta & 9,85 & 11,35 & 180,6 & 85,2 & $1.778,9$ & 967,0 & $2.745,9$ \\
\hline 2011 & Kombi Standard & 6,95 & 7,15 & 107,7 & 435,0 & 748,5 & $3.110,3$ & $3.858,8$ \\
\hline 2011 & Palio Weekend ELX & 8,79 & 9,90 & $2.223,5$ & $4.213,8$ & $19.544,6$ & $41.716,6$ & $61.261,2$ \\
\hline 2011 & Parati 1.6 Plus & 11,32 & 16,55 & & 301,7 & & $4.993,1$ & $4.993,1$ \\
\hline 2011 & Strada Fire & 8,9 & 10,10 & $2.060,9$ & $1.434,6$ & $18.445,1$ & $14.489,5$ & $32.934,5$ \\
\hline 2012 & Dobló Attractiv & 6,15 & 9,05 & 44,3 & 319,7 & 272,4 & $2.893,3$ & $3.165,7$ \\
\hline 2012 & Palio Weekend ELX & 8,79 & 9,90 & 591,4 & $1.853,1$ & $5.198,4$ & $18.345,7$ & $23.544,1$ \\
\hline 2012 & Strada Fire & 8,95 & 10,10 & 558,2 & $1.703,1$ & $4.995,9$ & $17.201,3$ & $22.197,2$ \\
\hline 2012 & Strada Locker Adv. & 7,90 & 8,95 & 422,6 & $2.885,2$ & $3.338,5$ & $25.822,5$ & $29.161,1$ \\
\hline 2012 & Uno Attractive & 8,95 & 11,20 & $1.436,6$ & $4.498,5$ & $12.857,6$ & $50.383,2$ & $63.240,8$ \\
\hline
\end{tabular}

\section{Abastecimento}

A quantidade de combustível abastecido coletada na base de dados e estimada no uso exclusivo do etanol (Equação 7), por ano de fabricação do modelo. Assim, notou-se aumento líquido de $26 \%$ na quantidade de abastecimento necessária (Tabela 6).

Tabela 6. Total de combustíveis utilizado por ano de fabricação

\begin{tabular}{|c|c|c|c|c|c|}
\hline \multirow{3}{*}{$\begin{array}{l}\text { Ano da } \\
\text { frota }\end{array}$} & \multicolumn{4}{|c|}{$\begin{array}{l}\text { Cenário } 1 \\
\text { Consumo real em } 2014\end{array}$} & \multirow{3}{*}{$\begin{array}{c}\text { Cenário } 2 \\
\text { Consumo estimado } \\
\text { Etanol hidratado }\end{array}$} \\
\hline & \multirow{2}{*}{$\begin{array}{c}\text { Etanol } \\
\text { hidratado }\end{array}$} & \multicolumn{3}{|c|}{ Gasolina Tipo C } & \\
\hline & & Total & Gasolina Tipo A (75\%) & Etanol Anidro (25\%) & \\
\hline 2006 & 18.235 & 85.576 & 64.182 & 21.394 & 144.085 \\
\hline 2007 & 34.504 & 206.349 & 154.762 & 51.587 & 312.583 \\
\hline 2008 & & 2.624 & 1.968 & 656 & 3.848 \\
\hline 2009 & 16.456 & 108.889 & 81.667 & 27.222 & 168.444 \\
\hline 2010 & & 7.092 & 5.319 & 1.773 & 9.919 \\
\hline 2011 & 32.357 & 124.951 & 93.713 & 31.238 & 181.536 \\
\hline 2012 & 20.996 & 144.308 & 108.231 & 36.077 & 191.723 \\
\hline 2014 & 44 & 156 & 117 & 39 & 270 \\
\hline Totais & 122.593 & 679.945 & 509.959 & 169.986 & 1.012 .408 \\
\hline
\end{tabular}

\section{Valores pagos nos abastecimentos}

A Tabela 7 apresenta os valores calculados de pagamento dos abastecimentos por categorias 
no mês de janeiro de 2014, a título de exemplo. Os mesmos cálculos foram realizados por mês para o ano do estudo. Assim, com a quantidade de combustível necessária por mês e com os valores mensais do etanol (Tabela 3), obtêm-se as estimativas dos valores pagos caso a opção de abastecimento fosse exclusivamente com etanol.

Tabela 7. Exemplos de cálculos de valores pagos por categorias no mês de janeiro/2014

\begin{tabular}{|c|c|c|c|c|c|c|}
\hline \multirow[b]{2}{*}{ Ano } & \multirow[b]{2}{*}{ Modelo } & \multicolumn{2}{|c|}{ Abastecimento (L) } & \multicolumn{3}{|c|}{ Valores pagos (R\$) } \\
\hline & & Etanol & Gasolina & $\begin{array}{c}\text { Etanol } \\
(\mathrm{R} \$ 2,046 / \mathrm{L})\end{array}$ & $\begin{array}{c}\text { Gasolina } \\
(\mathrm{R} \$ 2,994 / \mathrm{L})\end{array}$ & Total \\
\hline 2006 & Kombi Furgão & & 62,3 & & 186,5 & 186,53 \\
\hline 2006 & Saveiro & $3.085,9$ & $6.444,7$ & $6.313,8$ & $19.295,4$ & $25.609,18$ \\
\hline 2007 & Kangoo AUT Express & 198,5 & 810,3 & 406,1 & $2.426,0$ & $2.832,17$ \\
\hline 2007 & Kangoo RL 1.6 Expr. & 299,5 & $2.909,2$ & 612,8 & $8.710,1$ & $9.322,92$ \\
\hline 2007 & Kombi Standard & & 57,4 & & 171,9 & 171,86 \\
\hline 2007 & Uno Mille Fire & $1.248,5$ & $3.883,4$ & $2.554,4$ & $11.626,9$ & $14.181,33$ \\
\hline 2007 & Parati 1.6 Plus & 834,1 & $1.149,1$ & $1.706,6$ & $3.440,4$ & $5.146,97$ \\
\hline 2007 & Saveiro & $2.522,0$ & $4.606,6$ & $5.160,0$ & $13.792,2$ & $18.952,17$ \\
\hline 2008 & Gol Geração 5 & & 84,2 & & 252,1 & 252,09 \\
\hline 2009 & Dobló Cargo & 122,5 & $1.227,0$ & 250,6 & $3.673,6$ & $3.924,27$ \\
\hline 2009 & Gol Geração 4 & $1.170,1$ & $2.002,8$ & $2.394,0$ & $5.996,4$ & $8.390,41$ \\
\hline 2009 & Parati 1.6 Plus & 429,1 & $1.070,1$ & 877,9 & $3.203,9$ & $4.081,82$ \\
\hline 2009 & Saveiro & $1.178,3$ & $3.410,4$ & $2.410,8$ & $10.210,7$ & $12.621,54$ \\
\hline 2010 & Dobló Cargo & & 271,2 & & 812,0 & 811,97 \\
\hline 2010 & Parati 1.6 Plus & & 532,8 & & $1.595,2$ & $1.595,20$ \\
\hline 2011 & Dobló Attractiv & & 566,3 & & $1.695,5$ & $1.695,50$ \\
\hline 2011 & Dobló Cargo & 49,5 & $1.195,5$ & 101,3 & $3.579,3$ & $3.680,60$ \\
\hline 2011 & Fiesta & 180,6 & 85,2 & 369,5 & 255,1 & 624,60 \\
\hline 2011 & Kombi Standard & 107,7 & 435,0 & 220,4 & $1.302,4$ & $1.522,74$ \\
\hline 2011 & Palio Week ELX & $2.223,5$ & $4.213,8$ & $4.549,3$ & $12.616,1$ & $17.165,40$ \\
\hline 2011 & Parati 1.6 Plus & & 301,7 & & 903,3 & 903,29 \\
\hline 2011 & Strada Fire & $2.060,9$ & $1.434,6$ & $4.216,6$ & $4.295,2$ & $8.511,79$ \\
\hline 2012 & Dobló Attractiv & 44,3 & 319,7 & 90,6 & 957,2 & $1.047,82$ \\
\hline 2012 & Palio Week ELX & 591,4 & $1.853,1$ & $1.210,0$ & $5.548,2$ & $6.758,19$ \\
\hline 2012 & Strada Fire & 558,2 & $1.703,1$ & $1.142,1$ & $5.099,1$ & $6.241,16$ \\
\hline 2012 & Strada Locker Advent & 422,6 & $2.885,2$ & 864,6 & $8.638,3$ & $9.502,93$ \\
\hline 2012 & Uno Attractive Evo & $1.436,6$ & $4.498,5$ & $2.939,3$ & $13.468,5$ & $16.407,79$ \\
\hline
\end{tabular}

A Tabela 8 apresenta a diferença de valores a serem pagos para cada $100 \mathrm{~km}$ rodados entre os dois cenários. Os valores negativos demonstram que não há economia, o que está de acordo com a equação comparativa de vantagem econômica da opção etanol (Equação 8), mostrando a vantagem da adoção desse combustível quando a relação entre os rendimentos dos motores de determinado veículo com cada combustível for maior que a relação entre os preços dos dois combustíveis no momento do abastecimento.

No caso estudado, 99 pontos foram negativos, de um total de 348, porém a representatividade desses casos foi baixa. A média dos valores positivos indicativos de economia foi de $\mathrm{R} \$ 4,97$ a cada $100 \mathrm{~km}$, enquanto a média dos valores negativos foi de apenas -R \$ 0,55 para a mesma distância. Portanto, no geral, a vantagem foi do biocombustível. O comparativo mostrou uma economia de $8,5 \%$ nos recursos financeiros com a adoção exclusiva do etanol nos abastecimentos.

\section{Emissões de gases de efeito estufa}

As emissões totais estimadas em $\mathrm{CO}_{2}$ equivalente caso os veículos do estudo fossem abastecidos com etanol foi de 1.522,9 toneladas métricas. Isso equivale a uma redução de $6,1 \%$ do montante realmente emitido utilizando predominantemente o combustível fóssil. Contudo, $\mathrm{O} \mathrm{CO}_{2}$ emitido referente à biomassa correspondeu ao carbono estocado a partir da retirada da atmosfera pelo processo de fotossíntese no cultivo da cana-de-açúcar (SANTOS, 2008). Considerando isso, temos redução no balanço final das emissões de $\mathrm{CO}_{2}$ eq de 1.183,8 toneladas. O que corresponderia a uma redução de $96 \%$. Esses valores são apresentados na Tabela 9. 
Tabela 8. Demonstrativo de economia entre o cenário utilizando bi-combustível e o cenário estimado utilizando somente etanol, por modelo de veículo, mês a mês durante 2014 (em $\mathrm{R} \$ / 100 \mathrm{~km}$ )

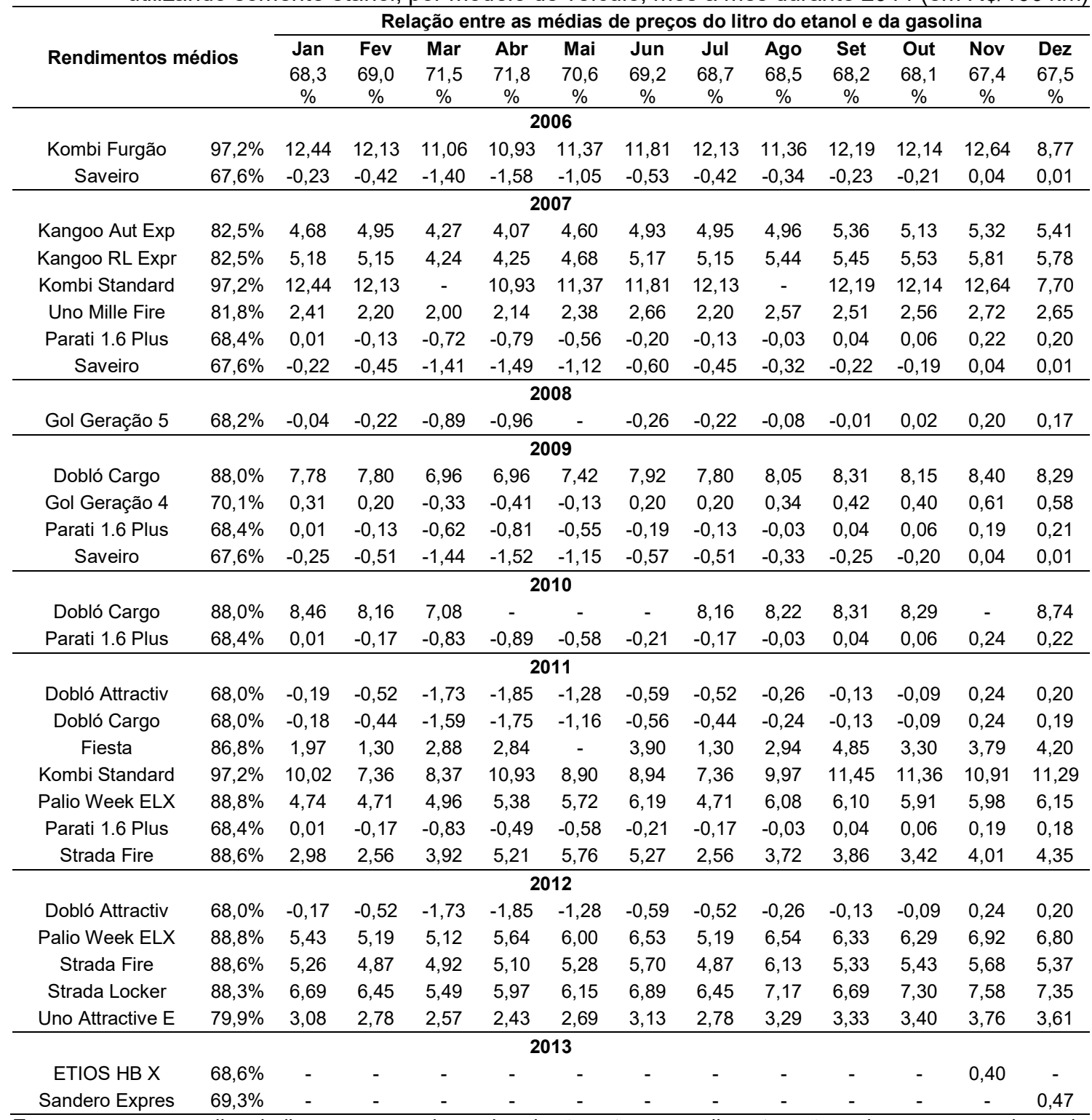

Em que: cores vermelhas indicam que os valores da relação entre os rendimentos são maiores que os valores da relação dos preços.

\section{DISCUSSÃO E CONCLUSÃO}

Por meio dos dados de abastecimento de uma frota de 508 veículos leves tipo flex-fuel, de modelos que receberam selo de eficiência do INMETRO, por meio do Programa Brasileiro de Etiquetagem Veicular e a metodologia Botton-Up e a ferramenta GHG Protocol Brasil, calculou-se o valor total pago de abastecimento e a quantidade de emissões de gases de efeito estufa para dois cenários: a) histórico, com o abastecimento utilizando-se tanto gasolina quanto etanol; e b) estimado, com o abastecimento exclusivo de etanol necessário para cobrir a mesma distância. Assim, mesmo com um volume bruto de abastecimento $26 \%$ maior do etanol, a opção da escolha exclusiva pelo biocombustível demonstrou uma economia em torno de 10\%. Essa percentagem, porém, foi diferente para cada modelo de veículo.

A diferença entre o preço ao consumidor dos dois combustíveis foi outra variável importante. Este trabalho indicou a comparação entre a razão de eficiências aferidas pelo INMETRO em conjunto 
à razão entre os preços dos combustíveis no momento do abastecimento, sendo uma forma de obter a maior economia, caso seja essa a única variável de interesse. Como exemplo, pode-se utilizar a média da relação entre a eficiência da utilização do etanol e da gasolina nos veículos, sendo de $78,7 \%$ e superior os $70 \%$ da linha de corte. Isso indica que, em média, se a relação entre o preço do etanol e da gasolina for menor que esse resultado, é válida a escolha da opção não fóssil.

Tabela 9. Emissões de gases de efeito estufa, em toneladas $\mathrm{CO}_{2 \mathrm{eq}}$

\begin{tabular}{cccc}
\hline Abastecimento & GEE emitidos (CO $\left.\mathbf{C O}_{\text {eq }}\right)$ & Cenário 1 & Cenário 2 \\
\hline \multirow{3}{*}{ Etanol Hidratado } & $\mathrm{CO} 2$ biomassa & 178,6 & 1475,1 \\
& $\mathrm{CH}$ 4 etanol & 0,8 & 6,4 \\
& $\mathrm{~N} 2 \mathrm{O}$ etanol & 5,0 & 41,4 \\
& Total CO2 equivalente & 5,8 & 47,8 \\
\hline \multirow{3}{*}{ Gasolina Tipo C } & $\mathrm{CO}$ 2 biomassa (etanol anidro) & 259,4 & \\
& $\mathrm{CO} 2$ direto (gasolina) & $1.128,0$ & \\
& $\mathrm{CH} 4$ & 2,9 & \\
& $\mathrm{~N} 2 \mathrm{O}$ & 47,1 & \\
\hline \multirow{2}{*}{ Total } & Total CO2 equivalente & $1.178,0$ & \\
& $\mathrm{CO} 2$ biomassa & 438,0 & $1.475,1$ \\
& Total CO2 equivalente & $1.183,8$ & 47,8 \\
& Total & $\mathbf{1 . 6 2 1 . 9}$ & $\mathbf{1 . 5 2 2 , 9}$ \\
\hline
\end{tabular}

Contudo, o ganho ambiental também deve ser considerado e, mesmo sem considerar o balanço energético na produção do etanol a partir do bagaço da cana-de-açúcar, há vantagem no uso do biocombustível. Com isso, esse trabalho apontou para uma diminuição de emissões de GEE na casa dos $6 \%$, somente considerando a queima do combustível, sendo uma redução de impacto se estendida a toda a frota do estado e, por extensão, do país, além de contribuir para o aumento da participação dos biocombustíveis na matriz energética, principalmente no setor de transportes, ainda muito dependente da opção fóssil.

Apesar da vantagem econômica da opção mais sustentável nas condições atuais de mercado, a relação entre os preços dos dois combustíveis apresenta muita variação, uma vez que depende das políticas públicas, do mercado internacional, das safras da cana-de-açúcar, da concorrência do plantio de outras culturas pela mesma área, além de outros fatores.

Dessa maneira, recomenda-se a adoção da exploração dos ganhos econômicos indiretos da escolha do biocombustível, como, por exemplo, o ganho com a imagem e a reputação empresarial. As opções de transportes com menores emissores de GEE contribuem na obtenção de certificações verdes, como o selo americano LEED e a francesa AQUA - HQE.

Seja qual for a política de comunicação da empresa, a opção por alternativas mais sustentáveis tem um potencial de valoração da marca e deve ser explorada, pois, além de servir como potencial competitivo, incentiva toda a cadeia de produção e comercialização a adotar opções semelhantes, contribuindo para uma melhora geral na qualidade ambiental do planeta.

\section{REFERÊNCIAS}

ANP - Agência Nacional do Petróleo, Gás Natural e Combustível. Evolução do mercado de combustíveis e derivados: 2000-2012. Rio de Janeiro, 2013. Disponível em: <http://www.anp.gov.br/?id=711> Acesso em: 15/08/2015.

ANP - Agência Nacional do Petróleo, Gás Natural e Combustível. Boletim anual de preços - preços do petróleo, gás natural e combustíveis nos mercados nacional e internacional. Rio de Janeiro: ANP, 2014. Disponível em: <http://www.anp.gov.br/?dw=73131> Acesso em: 15/08/2015.

ANP - Agência Nacional do Petróleo, Gás Natural e Combustível. Relatório de defesa da concorrência, gerado pelo sistema de levantamento de preços. 2015. Disponível em:<http://www.anp.gov.br/preco/prc/resumo_por_estado_index.asp> Acesso em: 15/08/2015.

ANDRADE, A. L. C.; MATTEI, L. Consumo energético e emissões de $\mathrm{CO}_{2}$ : uma análise do setor de transportes brasileiro. In: IX Encontro da Sociedade Brasileira de Economia Ecológica, Anais... Brasília: 2011. 
COLLARES, D. G. Embrapa agroenergia apresenta balanço energético do etanol. Disponível em: <http://www.yete.com.br/yete/index.php?\&view=article\&id=72> Acesso em: 12/08/2015.

CONAMA - Conselho Nacional do Meio Ambiente. Resolução Conama $\mathbf{n}^{\circ} 15$ de 13 de dezembro de 1995. 1995. Disponível em: <http://www.mma.gov.br/port/conama/legislacao/CONAMA_RES_CONS_ 1995_01 5.pdf> Acesso em: 13/08/2015.

CIMA - Conselho Interministerial do Açúcar e do Álcool. Resolução CIMA n 1 de 4 de março de 2015. 2015. Disponível em: <http://www.jusbrasil.com.br/diarios/87237712/dou-secao-1-06-03-2015pg-17> Acesso em: 13/08/2015.

FILHO, A. A. V.; RAMOS, P. Proálcool e evidências de concentração na produção e processamento de cana de açúcar. Informações Econômicas, v. 36, n. 7, 2006.

INMETRO - Instituto Nacional de Metrologia, Qualidade e Tecnologia. 2015. Tabelas PBE Veicular. Disponível em: <http://www.inmetro.gov.br/consumidor/tabelas_pbe_veicular.asp> Acesso em: $15 / 08 / 2015$.

INMETRO - Instituto Nacional de Metrologia, Qualidade e Tecnologia. Metodologia para divulgação de dados de consumo veicular. 2010. Disponível em: <https://http://www.inmetro.gov.br/consumidor/ pbe/Metodologia_Consumo_Veicular.pdf> Acesso em: 15/08/2015.

IPCC - Intergovernmental Panel on Climate Change. Fourth assessment report: climate change 2007. 2007. Disponível em <http://www.ipcc.ch/publications_and_data/ar4/wg1/en/ts.html>. Acesso em $15 / 08 / 2015$.

IPCC - Intergovernmental Panel on Climate Change. Climate change 2014 synthesis report fifth assessment report. 2014. Disponível em: <http://ar5-syr.ipcc.ch/> Acesso em: 15/08/2015.

MAPA - Ministro de Estado da Agricultura, Pecuária e Abastecimento. Portaria MAPA n 105 de 28 de fevereiro de 2013. 2013.

SANTOS, A. C. A Influência do uso do etanol combustível nas emissões dos gases de efeito estufa nos motores ciclo Otto, 109 p. 2008. Dissertação (Mestrado em Engenharia de Processos Químicos e Bioquímicos) - Escola de Engenharia Mauá, Centro Universitário do Instituto Mauá de Tecnologia, São Caetano do Sul.

PROGRAMA BRASILEIRO GHG PROTOCOL. Ferramenta de cálculo. 2014. Disponível em: <http://www.ghgprotocolbrasil.com.br/ferramenta-de-calculo> Acesso em: 15 /08/2015. 